\title{
Personal health records
}

\author{
Sir Douglas Black Consulting Editor, Journal of Medical Ethics
}

In countries in which a general practitioner service has been maintained, each citizen should have a personal health record maintained by his family doctor, and transferred when he changes his doctor. These records are generally in written form, in the custody of the doctor; and it is understood between patient and doctor that the information in the record is confidential. Much of what is in the record has been supplied by the patient himself, but on a basis of trust that the information so supplied will be used in the patient's health care, and not for other purposes; but there may also be in the record other types of information, for example that supplied by other doctors in consultation, or by parents in respect of children. Such additional material should also be assumed to lie within the context of the health care of that particular patient - it may therefore be properly divulged, on a 'need to know' basis, to other participants in what is commonly a 'health care team'. But such 'implicit consent', stemming from the understanding on which the information was originally given, cannot properly be extended outside the limits of personal clinical care, without giving specific consideration to the patient's entitlement to autonomy.

For some ethicists, the right to autonomy is absolute, so that perhaps even the transfer of information in the course of clinical care should be validated by prior explicit consent, and not by tacit understanding that the doctor is there to help the patient, as best he can. Given that consent is only valid when it is 'informed', there seem to me to be pragmatic difficulties in a position which demands prior justification of each transfer of information in the clinical process, not all of which is leisurely. But apart from what would at one time have been a selfish wish to facilitate the clinical process, I see two further difficulties in an absolutist position on autonomy. The first of these relates to conflict with the 'public interest', the second to conflict with 'beneficence'.

perhaps the clearest example of conflict with the public interest arises when information obtained either from history or examination of a patient makes it probable that he has been engaged in terrorism or other crime of comparable severity. Less dramatic, it is clear that in an organised health service it is important for the efficiency of the service that health information on individuals should be made available for the proper activities of management, and of the 'public health function', including 'medical audit' which would be quite misleading without information on the clinical state of those entering the system which is being 'audited'. Apart possibly from exceptions on a 'law and order' basis (and perhaps not even then), autonomy should not be breached on a 'catch-all' basis, but only on demonstrable necessity. It should not, for example, be breached as a matter of accounting convenience.

In relation to conflict with beneficence, the assumption which legitimates a limited trespass on autonomy is this, that a man or woman of goodwill may as an act of grace sanction the use of their personal health information for purposes which are neither an integral part of their own health care, nor forced upon them by authority. On an absolutist view of autonomy, they might be influenced against such actions, to the prejudice of medical research. As a matter of experience, consent to participate in medical research, even at some personal cost, is freely given when the nature and purpose of the research are explained, and duress is explicitly disclaimed. In other words, there is a general willingness to set beneficence above autonomy in a clinical setting. That beneficence can be elicited by appeal does not imply that it can be assumed without further consideration; and some professional groups explicity take the view that there can be no access to personal health records without explicit informed prior consent. Rigorous adherence to that position would spell the end of epidemiological research, from which great benefits have flowed. For that research of its very nature requires large numbers, making individual appeal for consent difficult, even impracticable, and in the case of research based on death certificates frankly impossible. Given that epidemiological research is of its nature observational and not interventive, and that publication does not allow the identification of any individual whose records are used, the possibilities of harm seem remote - even so, they should not be left solely to the judgement of an isolated investigator with a stake in doing the work. Possible safeguards include the consent of the custodian of records, and the scrutiny of an independent ethics committee; but the real and ultimate safeguard is the tradition of professional 
confidentiality which goes back to Hippocrates. This obligation should be extended by contract or other means to all who have access to personal health records. A detailed and informative discussion of the ethical issues raised by epidemiological studies has been issued by the Council for International Organisations of Medical Sciences (CIOMS) (1).

These principles should apply to all health records, whether 'manual' or 'mechanically processed'. By what is likely to be a temporary anomaly, the operation of the Data Protection Act in the UK extends only to mechanically processed records, though a presumption of confidentiality must also exist for the presently more numerous manual records. There is obviously considerable anxiety among legislators and the public, about insecurity of information held mechanically - anxiety which to some extent has spilt over to health records from other areas such as financial credit, where the numerical power and speed of mechanical data-processing are as impressive as the risks that not all the inputs to the system may be accurate. This last fear has created the appetite for 'subject access', ie the right of the 'subject' to gain 'sight' of his record, if only for the purpose of checking its accuracy. This right has now been legally conceded, with some safeguards to protect those parts of the record not contributed by the patient himself, and also to avoid the needless alarm which might arise from seeing a list of possible diagnoses which were later excluded, but which had been distinctly possible at an earlier stage of investigation, and were thus important parts of the record.

Partly arising out of the debate on subject access, it has become recognised that in some specific areas of practice, for example the antenatal clinic, there may be an advantage in giving the patient custody of her own record; nor would it be difficult for her to better the 'availability-rate' of 60 per cent in a (bad) manual system. This type of arrangement may well become more general, with the increasing development and use of 'patient data cards' (PDCs), which have been studied by a Working Group of the European Commission (2). PDCs are of different types, based on magnetic strips, lasers, or on integrated circuits - the so-called 'smart card'. They have in common the option of having part of the information visible, or 'external', for identification purposes; and the rest 'internal' or 'closed', accessible only through a template system, and thus capable of restriction to designated people. The restriction can operate at two levels, the first allowing access to 'administrative staff and medical personnel', the second only to 'designated health care professionals'.

Without claiming to have extracted every nuance from the Eurospeak in which this document is written, I have been surprised by two apparent omissions - the lack of any provision for the subject to gain access to his own health information; and there is little, if anything, on the custody and safe-keeping of the PDC. It is not unknown for cards to be lost or mislaid, whether by patients or in record stores; and any established sytem must surely include provision for duplicate cards, and for precautions to prevent improper access to any held centrally. Nevertheless, the statements of both general principles (pp 150-154) and of principles specific to PDCs (pp 155-159) give useful information on the present situation, as seen after a European study in depth.

Sir Douglas Black, MD, FRCP, is Emeritus Professor of Medicine, University of Manchester and a Past President of the Royal College of Physicians and of the British Medical Association. He is a Consulting Editor of the Journal of Medical Ethics.

\section{References}

(1) CIOMS. International guidelines for ethical review of epidemiological studies. Geneva: CIOMS, 1991.

(2) AIM Patient Data Card Working Group. AIM Report on assessment of the needs and organisational impact of the patient data cards. Brussels: Commission of the European Communities, 1990. 